\title{
Rare case of round blue cell pituitary tumour with probable hypothalamic involvement
}

S. Zhyzhneuskaya ${ }^{1}$, A. Mitchell' ${ }^{2}$, M. Ganguri', J. Abbas', S.Biswas ${ }^{2}$, P.Perros ${ }^{2}$, P.Kane ${ }^{1}$, S.Ashwell', S. Nag', R. Mukhtar', S. Jones', Prof. R. Bilous', V. Arutchelvam¹

${ }^{1}$ Departments of Endocrinology, JCUH, Middlesbrough, ${ }^{2} \mathrm{RVI}$, University of Newcastle, UK
South Tees Hospitals NHS NHS Foundation Trust

\section{Introduction}

We submit a rare presentation of round blue cell pituitary tumour complicated by cranial diabetes insipidus following

transphenoidal surgery.

\section{Case history}

- 47 year old lady

- Severe headache

- $10 \mathrm{~mm}$ non functioning pituitary macro adenoma.

- Initial plan for conservative management - revised as she developed sixth cranial nerve palsy, bi temporal hemianopia and rapidly enlarging pituitary tumour to $20 \times 18 \times 19 \mathrm{~mm}$.

- Urgent Transphenoidal pituitary surgery

- Repeat surgery in 2 weeks as deteriorated.

- Pituitary tumour was found to be of fibrous consistency

- Histology confirmed poorly differentiated round blue cell tumour with mitotic index 50\%, positive for CD99 and CD56, cytogenetically unclassified.

- INI-1 negative

- Genetic studies awaited.

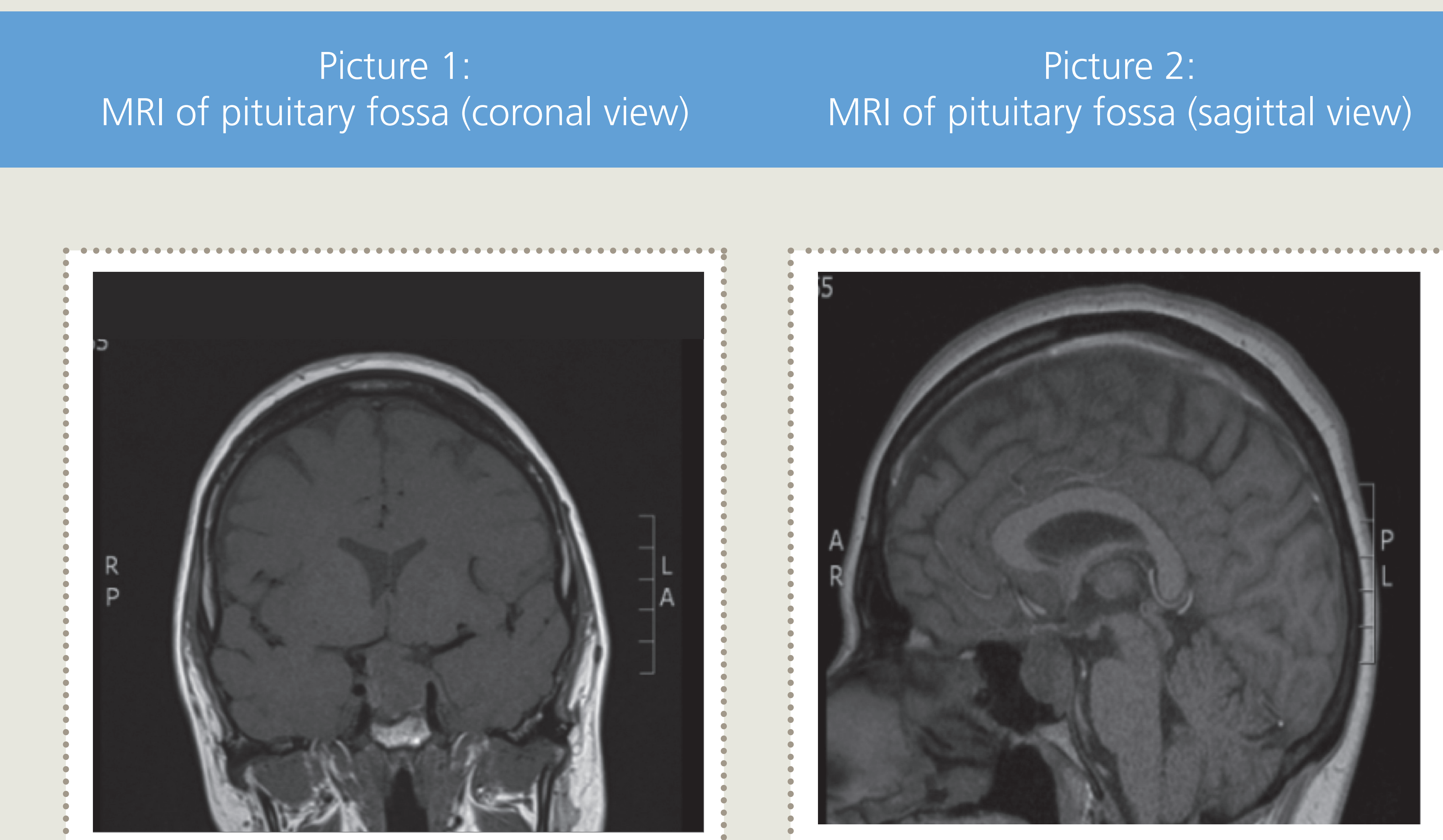

\section{Management and Discussion}

- On 1st post-op day, she developed polyuria and polydipsia, diagnosed with cranial DI and commenced on DDAVP.

- Next day she had a generalised seizure caused by rapidly developing hyponatraemia, managed with strict fluid balance and a fluid restriction of $1.5 \mathrm{~L}$ daily with dynamic management DDAVP dose.

- Developed excessive thirst despite normal serum sodium and urine osmolality raising the possibility of hypothalamic thirst centre being affected by the aggressive pituitary tumour.

- Chemotherapy started.

- Patient had some improvement in her vision after 2 cycles of chemotherapy with overall prognosis very poor.

- Passed away after 4 weeks.

\section{Conclusion}

We presented this case to illustrate a rare, aggressive pituitary malignancy which possibly has invaded the hypothalamus causing disruption to thirst mechanism in addition to causing CDI.

\section{Histology images}

Haematoxylin and eosin, original magnification x 40.Cells with scanty cytoplasm and large vesicular nuclei with prominent nucleoli. Haematoxylin and eosin, original magnification x40.Cells with scanty cytoplasm and large vesicular nuclei with prominent nucleoli.
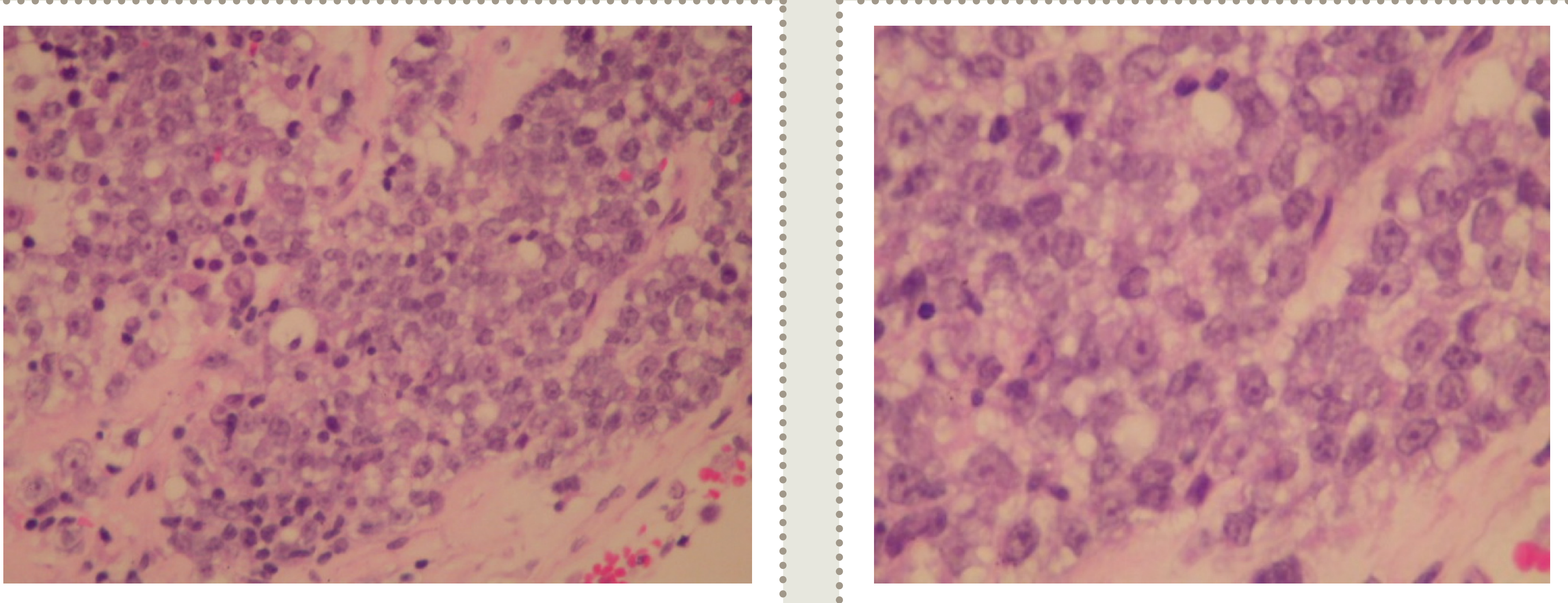

Contact details: vijayaraman.arutchelvam@stees.nhs.uk 